\title{
Aspectos gerais do tratamento de cessação ao tabagismo: Uma revisão de
} literatura

\author{
Joaquim Horácio de Araújo Neto
}

Márcia Jordana de Araújo

Ana Aline Kércia de Araújo

Ana Flavia Vasconselos de Paula

Rafaelly Maria Pinheiro Siqueira

\section{Registro DOI: http://dx.doi.org/10.22280/revintervol12ed2.446}

\begin{abstract}
Resumo
O objetivo deste trabalho foi realizar um levantamento bibliográfico sobre o tratamento de cessação do tabagismo procurando enfatizar as políticas, as medicações utilizadas, e outras informações inerentes ao consumo de produtos derivados do tabaco. Para tal, realizou-se um levantamento bibliográfico nas bases de dados SciELO, Medline e PubMed, com trabalhos publicados entre os anos de 2007 e 2018. Os resultados demonstram que no Brasil possui muitas políticas relacionadas que estimulam o tratamento de cessação, oferecendo o serviço gratuito tendo os adesivos transdérmicos como terapia farmacológica de primeira escolha. $\mathrm{O}$ farmacêutico desempenha importante função nas orientações técnicas. Portanto, torna-se importante o conhecimento sobre os aspectos gerais do tabagismo, pois, por se tratar de uma política em construção, ainda são escassas as produções sobre os limites e potencialidades dessa política no Brasil.
\end{abstract}

Palavras-chave: Tabagismo. Cessação Tabágica. Tabaco.

General aspects of the smoking cessation treatment: A review of the literature

\begin{abstract}
The objective of this study was to conduct a literature review on the treatment of smoking cessation, emphasizing the policies, medications used, and other information related to the consumption of tobacco products. A bibliographic survey was carried out in the SciELO, Medline and PubMed databases, with papers published between the years 2007 and 2018. In Brazil, there are many related policies that stimulate cessation treatment, offering free service having the transdermal patches as a first-line pharmacological therapy. The pharmacist plays an important role in the technical guidelines. Therefore, knowledge about the general aspects




\section{Revinter}

of smoking becomes important, since it is a policy under construction, there are still few productions about the limits and potentialities of this policy in Brazil.Key-words: Maned wolf. Environmental enrichment. Behavior. Zoo. Enclosure.

Recebido em 05/06/2019 Aceito em 21/06/2019

\section{INTRODUÇÃO}

O tabagismo está classificado como uma das principais causas evitáveis de mortes no mundo. Dados epidemiológicos estimam que haja cerca de 1,1 bilhões de pessoas fumantes, fato esse que qualifica o tabagismo como uma doença pandêmica sendo representada como uma das principais ameaças à Saúde Pública. Aproximadamente $80 \%$ dessa população, habitam países subdesenvolvidos, sendo responsável por mais de 6 milhões de óbitos por ano relacionados com a prática habitual do uso direto do tabaco e 890 mil relacionados a exposição passiva ao fumo (WOLD HEALTH ORGANIZATION, 2018).

No Brasil, cerca de 24,6 milhões de pessoas são usuários do consumo de produtos derivados do tabaco, fumado ou não fumado, de uso diário ou ocasional. A prevalência entre os homens é de $21,6 \%$ totalizando 14,8 milhões de indivíduos. E entre as mulheres, a prevalência é de $13,1 \%$ o que equivale a 9,8 milhões de pessoas do sexo feminino (INSTITUTO NACIONAL DO CÂNCER, 2018).

Calcula-se que a cada ano mais de 156 mil pessoas venha a óbito por aparecimento de doenças associadas ao uso do tabaco, seja de forma ativa ou passiva (FOROUZANFAR et al., 2016).

Mais de 50 doenças estão relacionadas com os componentes tóxicos do tabaco. Dentre elas, as doenças respiratórias (DPOC, asma, bronquite, infecções respiratórias, enfisema pulmonar), doenças cardiovasculares (infarto agudo do miocárdio, trombose, acidente vascular cerebral, aneurisma e angina). E além destas, também estão relacionadas com os mais diversos tipos de câncer: câncer de pulmão, esôfago, faringe, laringe, estômago, rim, pâncreas, colo do útero e leucemias (SOARES et al., 2014).

Como consequências, os custos para o país somam $\mathrm{R} \$ 57$ bilhões por ano, sendo $\mathrm{R} \$$ 39,3 bilhões utilizados para assistência médica e tratamento, e R $\$ 17,7$ bilhões pela invalidez devido a perda da capacidade produtiva na população economicamente ativa (PINTO et al., 2017). 


\section{Revinter}

Desse modo, vem sendo articulado desde a década de 80 sob a concepção da promoção da saúde, as estratégias de controle do tabagismo no Brasil pelo Ministério da Saúde através do Instituto Nacional do Câncer - INCA, com o Programa Nacional de Controle do Tabagismo PCNT (COSTA et al., 2018).

A criação desse programa objetivou a redução dos consumidores de tabaco bem como as consequentes doenças associadas a ele. Suas intervenções ocorrem através de ações educativas e de atenção à saúde, de forma a oferecer suporte, alertar a população sobre os riscos e prevenir, assim, a iniciação do uso de derivados do tabaco, principalmente na população jovem e no tratamento do tabagismo no Sistema Único de Saúde (SUS), combatendo a dependência física e química (SILVA, 2014).

O grau de dependência é determinado pela equipe multiprofissional que respeita o protocolo de atendimento que é iniciado pelo acolhimento, preenchimento da ficha, história tabagística e encaminhamento para intervenções relacionadas a saúde mental e acompanhamento de atividades grupais, individuais e farmacológicos (INSTITUTO NACIONAL DO CÂNCER, 2015).

O presente trabalho teve como objetivo realizar um levantamento bibliográfico sobre o tratamento de cessação do tabagismo procurando enfatizar as políticas, as medicações utilizadas, e outras informações inerentes ao consumo de produtos derivados do tabaco.

\section{METODOLOGIA}

Este trabalho tratou-se de uma revisão bibliográfica, classificada como integrativa, pois foi elaborada e executada a partir de uma análise crítica das publicações escolhidas possibilitando a construção de conclusões oriunda de um estudo variado. Foram analisados trabalhos no período entre os anos de 2007 e 2018, incluindo artigos científicos, teses e dissertações, notícias relacionadas ao tabagismo e tratamento de cessação do consumo de produtos derivados do tabaco encontradas nas bases de dados SciELO, PubMed e MEDLINE. O critério de exclusão utilizado foram publicações anteriores ao ano de 2007. Sendo assim foram avaliados um total de 48 publicações para elaboração deste trabalho. Os descritores utilizados foram: tabagismo, cessação tabágica e tabaco.

\section{LEVANTAMENTO BIBLIOGRÁFICO}

Revinter, v. 12, n. 02, p. 66-83, jun. 2019. 


\section{Revinter}

\section{Aspectos gerais do tabagismo}

Sabe-se que tabagismo é uma denominação atribuída ao consumo de tabaco, que no universo científico recebeu o nome de Nicotiana tabacum como forma de homenagem ao embaixador da França na década de 80 do século XVI, Jean Nicot. O embaixador já desconfiava que o vegetal possuísse grande potencial medicinal. No século XVII, Catarina de Médicis, uma monarca da época, tomando conhecimento dos efeitos do tabaco, passou a fumar na tentativa de aliviar fortes dores de cabeça a que era acometida. Tendo resultados positivos, passou a indicar para amigos, familiares e assim sucessivamente. Desta forma, rapidamente o hábito do consumo de tabaco passou a ser largamente disseminada à nobreza do continente europeu (COSTA et al., 2018).

Em pouco tempo difundiu-se para os outros continentes sendo usados através da mastigação das folhas ou mediante a inalação na modalidade de rapé, charutos, cachimbos e em sua forma mais atual, o cigarro. Foram identificadas mais de 4.720 substâncias presentes na fumaça do cigarro. Mais de 200 delas, são consideradas tóxicas, como a terebintina, formol, amônia, naftalina, e 50 delas são cancerígenas. Além disso, encontra-se também a nicotina, princípio ativo responsável por causar dependência química. Tais circunstâncias estão relacionadas com ocorrência de mais de 50 doenças, destacando as mais incidentes: câncer de pulmão (90\%); bronquite crônica (85\%); enfisema pulmonar (85\%); infarto $(25 \%)$ e derrame cerebral (25\%) (INSTITUTO NACIONAL DO CÂNCER, 2015).

Atualmente, existe uma estimativa de que quase 5 milhões de pessoas morrem devido a complicações provocadas pelo uso do tabaco. Nesse ponto de vista, providências devem ser tomadas, caso contrário, indicadores apontam que no ano de 2020 esses índices de mortalidade relacionados com o tabagismo aumentem significativamente, podendo chegar aos impressionantes 8 milhões de mortes. O mais preocupante é que ao longo desse tempo houve um drástico aumento do fumo em países em desenvolvimento acometendo em especial os homens (PINTO; RIVIERE; BARDACH, 2015).

O tabagismo passivo também preocupa e é grave problema de saúde pública. Mundialmente, $40 \%$ das crianças, $35 \%$ das mulheres e $33 \%$ de homens que não fazem uso do tabaco, estão expostos à fumaça de seus derivados. Em paralelo, agrava essa situação, a estimativa de aproximadamente 600 mil mortes por ano, dentre os quais $26 \%$ em homens, $28 \%$ em crianças e 47\% em mulheres (OBERG, 2011). 
No Brasil, segundo Monteiro et al. (2007), houve uma redução da prevalência do tabagismo de $34,8 \%$ para $22,4 \%$ no período de 1989 a 2003. Estudos recentes feitos pelo INCA indicam que em 2018 a prevalência diminuiu para 10,2\%, o que representou uma redução importante quando comparada com os dados de 1989. O resultado positivo no controle do tabagismo no Brasil observado nas últimas décadas se deu pelas inúmeras ações desenvolvidas pelas políticas nacional do controle do tabaco.

\section{Políticas nacionais do controle do tabaco}

Estima-se que no Brasil, 156.200 pessoas venham ao óbito a cada ano por doenças ocasionadas pela pratica do fumo ativo ou passivo, mortes essas que são mais comuns em populações mais vulneráveis. Calcula-se que o custo, para o país, das consequências da prática tabagista aproxime-se de 57 bilhões por ano, configurando 39,5 bilhões com gastos da assistência médica e tratamento e 17,5 bilhões pela perda da produtividade e invalidez (PEREIRA et al., 2018).

Diante destes dados alarmantes, muitas políticas antitabagistas foram desenvolvidas. Em 1986 foi lançado no Brasil, através do Ministério da Saúde e o Instituto Nacional do Câncer (INCA), o Programa Nacional de Controle do Tabagismo (PNCT), que apresenta medidas de regulamentação e comércio de produtos provenientes do tabaco, envolvendo medidas preventivas para que as pessoas não venham iniciar o hábito de fumar, medidas de cessação ao tabagismo, e medidas de proteção à saúde dos usuários que não são fumantes (MENDES et al., 2016).

O Programa objetiva minimizar a prevalência de fumantes e de doenças relacionada ao consumo de derivados do tabaco, adotando um instrumento lógico de ações educativas, de comunicação, de atenção à saúde, em conjunto com a implantação de medidas legislativas e econômicas, para potencializar a prevenção à iniciação do tabagismo, principalmente entre os jovens. O PNCT articula a Rede de Tratamento do Tabagismo no SUS, as Campanhas e outras ações educativas e a Promoção de Ambientes Livres de Tabaco (BRASIL, 2015).

A lei Federal 9.294/96 de 15 de julho de 1996 amparou de certa forma as ações antitabagistas, proibindo as propagandas que tivessem um conteúdo com potencial capacidade de indução ao consumo de cigarros e derivados do tabaco. Além disso, embora seja lícito, o 


\section{Revinter}

tabaco passou a ser visto como uma droga com alto poder prejudicial para a saúde, devido as suas inúmeras substâncias tóxicas (COSTA et al., 2018).

As políticas brasileiras para o controle do tabagismo tiveram avanços nos últimos vinte anos. Algumas ações contribuem de forma eficaz para a redução de fumantes, podendo citá-las como exemplo o fortalecimento de campanhas educativas, aumento de impostos sobre os cigarros, proibição do fumo em ambientes fechados e à criação de programas de apoio aos fumantes que pretendem abandonar o vício (MORAES, 2015).

O interesse e a ativa participação popular também se tornam essencial para o desenvolvimento de novas políticas públicas de controle do tabagismo. Alguns trabalhos feitos através de redes sociais contribuíram para o aumento da conscientização sobre os danos associados ao tabagismo e das medidas eficientes para a prevenção do uso do tabaco e para a proteção da saúde dos brasileiros (PORTES et al., 2018).

Diante de Tantas ações realizadas para o combate do tabagismo, o maior adversário é a nicotina, devido sua elevada capacidade de dependência, promovendo intensa sensação de prazer diminuindo a ansiedade e o apetite. Essas sensações fortalece a atração dos usuários e principalmente para aqueles que usam o tabaco como forma de esquecer suas angústias e seus problemas de forma momentânea (RABAHI; ALCÂNTARA, 2015).

\section{Dependência ao tabagismo}

A Nicotina é a molécula responsável pelo desenvolvimento da dependência da droga. É um composto orgânico, e é o principal alcalóide presente no Nicotiana tabacum estando presente em todo o vegetal, sendo que 5\% de sua quantidade total está presente nas folhas (GOLAN et al., 2014).

A dependência a nicotina envolve uma série de fatores complexos, farmacológicos, comportamental e psicológico de cada pessoa. Está diretamente associada à quantidade diária de cigarros fumados, vulnerabilidade genética, tempo de exposição e condições socioeconômicas (AVEYARD et al., 2018).

Para Cappelletti (2017) a dependência a nicotina compreende o uso exagerado do fumo de maneira compulsiva, sem que o mesmo tenha a capacidade de parar ou ficar sem fumar por vontade própria por um tempo determinado, ou apresentar resistência para parar devido à síndrome de abstinência e desejo incontrolável para fumar. 
A Síndrome de abstinência e a dependência nicotínica estão classificadas como doença e tem critérios muito bem definidos, podendo ser avaliados pelo questionário de Fagerstron. Tem como sintomas fissuras, desconforto a nível gastrointestinal, bradicardia, ansiedade, insônia, euforia, aumento do apetite e, consequentemente, o aumento de peso (FERREIRA, 2014).

Além da dependência, outro fator que pode influenciar na cessação tabágica, é o ganho de peso. Estudos apontam que as pessoas que fumam pesam menos que pessoas que não fumam, e aumentam cerca de 4 a $5 \mathrm{Kg}$ nos primeiros 3 meses após a cessação do hábito de fumar devido ao considerado aumento no consumo de energia somando-se com a perca do efeito termogênico proporcionada pelo principio ativo. O ganho de peso, pode se tornar um desestimulador para as pessoas que pretendem parar de fumar (SILVA et al, 2016).

A nicotina entra no organismo através da inalação da fumaça, sendo absorvidas pelos alvéolos pulmonares e é transportada para o cérebro através do sangue. Aproximadamente $25 \%$ da droga inalada durante a prática fumígena consegue chegar ao sangue e atingir o encéfalo. Todo esse processo acontece em um período de 15 segundos (PUPULIM et al., 2015).

O tempo de meia-vida da nicotina é de aproximadamente duas horas. No cérebro, se liga em receptores colinérgicos nicotínicos denominados nAchR. Esses receptores (nAch) são os principais envolvidos na dependência. São formados por cadeias polipeptídicas com subunidades $\alpha 4$ e $\beta 2$. O contato da nicotina com esses receptores faz com que os mesmos sofram alterações conformacionais, promovendo a entrada de íons $\mathrm{Na}^{+}$e $\mathrm{Ca}^{+2}$. Esse evento impossibilita que a acetilcolina se ligue fisiologicamente. A passagem desses íons faz com que ocorra um fenômeno de despolarização que propaga o impulso nervoso até a região mesolímbica, onde se localiza o sistema de recompensa cerebral (GOLAN et al., 2014).

O sistema de recompensa cerebral consiste em neurônios dopaminérgicos na área tegmental ventral do mesencéfalo e em outras regiões cerebrais, como o núcleo acumbens e outras regiões estriatais ventrais. São nessas regiões onde ocorrem os fenômenos de tolerância e da vontade incontrolável em fumar em função da abstinência nicotínica.

A despolarização gerada nos neurônios da área tegumental do sistema de recompensa cerebral, resulta na liberação de dopamina. A dopamina é um neurotransmissor produzido a partir dos aminoácidos essenciais como a tirosina, que influencia diretamente nas reações de movimento, na emoção, no sono e etc (BRUNTON; CHABNER; KNOLLMANN, 2012). 


\section{Revinter}

Em pessoas tabagistas, a dopamina produz um efeito de reforço positivo. Estudos mais avançados em sobre a dependência, afirma que todas as drogas que induzem à dependência desencadeiam nos indivíduos os sintomas de euforia e prazer, agindo como reforçadoras positivas, sendo necessário um tratamento medicamentoso e psicológico para a cessação do vício (SILVA et al., 2016).

\section{Tratamento farmacológico para cessação do tabagismo}

A terapêutica farmacológica é muito importante devido ao seu percentual de sucesso ser superior aos demais métodos. O tratamento farmacológico está indicado para paciente que já apresentam uma dependência da nicotina classifica como moderada ou elevada, que fume mais de 10 cigarros, que apresente um resultado a partir de 5 no teste de Fagerstron e que tenha motivação suficiente para parar definitivamente do uso de produtos que contenha a nicotina (SILVA, 2015).

Os medicamentos utilizados para esse fim terapêutico são classificados como fármacos de primeira escolha por apresentarem um efeito mais eficaz sem muitas reações adversas, e os fármacos de segunda escolha por serem menos eficazes com mais efeitos adversos. Nos de primeira escolha estão o tratamento de substituição da nicotina (TSN), a vareniclina e a bupropiona. Dentre os fármacos de segunda escolha estão a nortriptilina e a clonidina (ROLLEMBERD et al., 2018).

\section{Fármacos de primeira escolha}

O tratamento de reposição de nicotina foi o primeiro método farmacológico utilizado para o tratamento do tabagismo. Existem diversos tipos de apresentação podendo estar nas seguintes formas farmacêuticas: adesivos transdérmicos, gomas de mascar, pastilhas, comprimidos, inalador ou spray nasal. Ambas as formas possibilitam uma maior eficácia no tratamento melhorando a adesão (MARTINS; CAPELA, 2010).

Com a administração da nicotina, seja por qual for a apresentação farmacêutica utilizada, matem o organismo com concentrações cada vez menores, minimizando os sintomas da abstinência e reduzindo a exposição do usuário aos efeitos nocivos das demais substâncias presentes no cigarro. A forma farmacêutica mais utilizada para o tratamento de reposição de 


\section{Revinter}

nicotina são os adesivos transdérmicos e as gomas de mascar. Os adesivos transdérmicos são fixados na pele liberando a nicotina na circulação por absorção cutânea. Na medida em que o tratamento avança, as doses de nicotina nos adesivos decrescem a cada dia, até chegar ao placebo (GARCIA, 2018).

O sistema publico de saúde distribui o medicamento de forma gratuita para os usuários que estão em tratamento de cessação do tabagismo. A posologia mais usual (Quadro 1), para pacientes muito dependente se aplica um adesivo a cada 24 horas iniciando com $21 \mathrm{mg} / \mathrm{dia}$ durante seis semanas, diminuindo para $14 \mathrm{mg} /$ dia durante mais duas semanas e depois $7 \mathrm{mg} / \mathrm{dia}$ durante uma ou duas semanas. Em fumante com um menor grau de dependência utilizam-se doses menores por 16 horas iniciando o tratamento com um adesivo de $15 \mathrm{mg} /$ dia durante seis semanas, reduzindo para $10 \mathrm{mg} /$ dia durante duas semanas e por fim, $5 \mathrm{mg} /$ dia por mais duas semanas (LEMES et al., 2017).

Quadro 1 - As principais apresentações, via de administração, dosagens e principais reações adversas das formulações de nicotina.

\begin{tabular}{|c|c|c|c|}
\hline Apresentação & Administração & Dosagens & Reações adversas \\
\hline Goma & Bucal & $2 \mathrm{mg} / 4 \mathrm{mg}$ & $\begin{array}{l}\text { Irritação da mucosa oral e } \\
\text { faríngea; dor e hipertrofia dos } \\
\text { músculos } \\
\text { indigestão e soluço. }\end{array}$ \\
\hline Pastilha & Bucal & $\begin{array}{c}1 \mathrm{mg} / 1,5 \mathrm{mg} / 2 \mathrm{mg} \\
/ 4 \mathrm{mg}\end{array}$ & $\begin{array}{l}\text { Irritação local; complicações } \\
\text { digestivas; soluço e aumento de } \\
\text { salivação. }\end{array}$ \\
\hline Comprimido & Sublingual & $2 \mathrm{mg} / 4 \mathrm{mg}$ & $\begin{array}{l}\text { Irritação local; boca seca; tosse e } \\
\text { soluço. }\end{array}$ \\
\hline $\begin{array}{c}\text { Adesivo } \\
\text { Transdérmico }\end{array}$ & Transdérmica & $\begin{array}{l}16 \mathrm{~h}: 15 / 10 / 5 \mathrm{mg} \\
24 \mathrm{~h}: 21 / 14 / 7 \mathrm{mg}\end{array}$ & $\begin{array}{l}\text { Irritação cutânea; prurido; } \\
\text { cefaleia e insônia e mialgias. }\end{array}$ \\
\hline
\end{tabular}

Fonte: LEMES et al., 2017.

Outro medicamento utilizado é a bupropiona. Trata-se de um medicamento da classe dos antidepressivos não tricíclicos que tem como mecanismo de ação a inibição da recaptação Revinter, v. 12, n. 02, p. 66-83, jun. 2019. 
de dopamina e de noradrenalina no neurônio pré-sináptico. Não se sabe ao certo, mas provavelmente sua ação nas vias dopaminérgicas central seja o mecanismo biológico responsável pela diminuição da vontade incontrolável de fumar nos pacientes que estão em tratamento com abstinência da nicotina (DANTAS et al., 2016).

No inicio do tratamento utiliza-se um comprimido de $150 \mathrm{mg}$ duas vezes ao dia dando um intervalo de oito horas entre uma dose e outra, devido seu efeito colateral ser provocar insônia sendo recomendado que a segunda dose coincida com o final da tarde ou inicio da noite. O tratamento com a bupropiona deve ser iniciado em pelo menos uma semana antes da abstinência para que seus níveis plasmáticos estejam constantes em concentrações ideais (ADHIKARI, 2014).

O tratamento deve continuar por três ou quatro meses com as doses de $300 \mathrm{mg} / \mathrm{dia}$ podendo ser associados com a terapia de reposição da nicotina em pacientes que tenha recaído e não conseguiram alcançar o objetivo terapêutico de abstinência total somente com a medicação de reposição nicotínica (ERVILHA, 2018).

Os efeitos colaterais da bupropiona mais relatados são: dor de cabeça, insônia boca seca, rash cutâneo e urticária, sendo esses muitas vezes os motivos pela interrupção do tratamento. Um dos benefícios relatados na literatura é seu efeito no ganho de peso que é comum em pacientes que deixam de fumar. Estudos mostram que pacientes tomando placebo ganharam mais peso que os pacientes que tomaram a medicação ativa (MOURA, 2014).

A vareniclina é um medicamento sintético produzido a partir da extração do alcaloide da planta Cytisus laburnum, tendo sua utilização no tratamento do tabagismo. Este medicamento possui um percentual de sucesso terapêutico bem maior que os demais medicamentos mencionados como de primeira escolha. (ERWIN; SLATON, 2014).

O mecanismo de ação da vareniclina consiste na ativação parcial dos receptores nicotínicos da acetilcolina, semelhante aos efeitos da provocado pela nicotina. Esse mecanismo ao ocupar parcialmente os receptores nicotínicos, reduz a vontade de fumar e aos poucos o usuário perde o hábito de consumir produtos fumígenos (MAGALHÃES; CAMARGO; SPINOSA, 2016).

O tratamento inicia-se de uma a duas semanas antes do dia programado para a abstinência. A posologia consiste em um aumento progressivo da dose para evitar possíveis eventos adversos por via oral: nos três primeiros dias usa-se $0,5 \mathrm{mg} / \mathrm{dia}$; do quarto ao sétimo dia usa-se $0,5 \mathrm{mg}$ duas vezes por dia; do oitavo dia até o fim do tratamento usa-se $1 \mathrm{mg}$ duas 


\section{Revinter}

vezes por dia. O tratamento normalmente tem duração de doze semanas, mas dependendo da condição clínica do paciente pode se estender por mais doze semanas (MARTINS; CAPELA, 2010).

O medicamento é contra indicado para mulheres gestantes ou que esteja na fase de aleitamento, e para menores de 18 anos. Geralmente os pacientes tem uma boa adesão terapêutica e tem poucas interações farmacológicas. As reações adversas mais relatadas são: náuseas, cefaleia, insônia, tontura e pesadelos. Foram relatados também alguns poucos casos de depressão e suicídio relacionados com o método terapêutico (BARBOZA, 2018).

\section{Fármacos de segunda escolha}

A nortriptilina é um fármaco que pertence a classe dos antidepressivos tricíclicos, utilizados para o tratamento da depressão. Atua inibindo a recaptação de noradrenalina e da serotonina. Não está bem elucidado o seu mecanismo na cessação do tabagismo, mas imaginase que esteja ligeiramente relacionado com a substuição dos efeitos da nicotina e na prevenção da depressão que é bem comum nos pacientes que estão em tratamento antitabagismo (CORONADO et al., 2018).

Normalmente, em pacientes que pretendem parar de fumar usa-se doses de 75 a 100 $\mathrm{mg} / \mathrm{dia}$. No início do tratamento usa-se $50 \mathrm{mg} / \mathrm{dia}$ ou gradualmente $25 \mathrm{mg}$ por três dias, dobrando a dose durante quatro dias, e em seguida chegando a $75 \mathrm{mg} / \mathrm{dia}$ durante 12 semanas. A administração das doses deve ser iniciada em pelo menos duas a três semanas antes do inicio da abstinência do tabaco (MARTINS, 2016).

As reações adversas mais relatadas são: distúrbios visuais, cefaleia, boca seca, distúrbios intestinais, tremores, fadiga e taquicardia (MARTINS; CAPELA, 2010).

A clonidina é um medicamento cujo seu mecanismo de ação está classificada farmacologicamente como agonista dos receptores $\alpha_{2}$ adrenérgico sendo geralmente utilizado por pacientes em tratamento de hipertensão e para redução dos sintomas da abstinência de álcool e opiáceos. No entanto alguns estudos relatam que a sua utilização vem sendo recorrente em paciente que estão fazendo a terapia antitabagismo (BARBOZA et al., 2016).

A dose utilizada para a terapia antifumo é de 0,15 a $0,75 \mathrm{mg}$ por dia pro um período que pode variar de três a dez semanas. Durante o período de tratamento é necessário reajustes de doses e uma observação ativa para evitar possíveis ocorrências de efeitos adversos. Na 


\section{Revinter}

literatura, os eventos adversos mais relatados são: insônia, depressão, tonturas, boca seca, fadiga e hipotensão ortostática. É um medicamento contra indicado na gravidez (BARAONA et al., 2017).

\section{Cuidado do profissional farmacêutico na cessação do tabagismo}

O farmacêutico em sua essência tem o dever de assegurar e zelar pela saúde pública atuando de forma a contribuir com a promoção da saúde e com a adoção de medidas educativas para conscientizar e orientar a população sobre bons hábitos. Também tem como dever assegurar uma correta orientação durante a dispensa de medicamentos assegurando que o usuário receba importantes informações sobre o uso dos medicamentos garantindo ao mesmo um tratamento com qualidade e seguro (SANTANA et al., 2018).

Seguindo essa linha de raciocínio, o profissional farmacêutico, cumprindo com seus deveres e obrigações devem estimular aos usuários fumantes, a cessação dessa pratica tão prejudicial para a saúde. Além de conhecer com profundidade sobre ações farmacológicas a respeito, pesquisas mostram que o farmacêutico é um profissional que está próximo aos pacientes, e que pode mais facilmente acompanhá-los ou aconselhá-los no tratamento e na melhor opção terapêutica (BENEZECH; CHAMPANET; ROUZAUD, 2018).

Alguns estudos enfatizam de forma positiva o aconselhamento farmacêutico para uma adesão satisfatória para o tratamento terapêutico da cessação da pratica tabágica comprovando que os pacientes que foram acompanhados por um profissional tiveram melhores resultados que os pacientes que não tiveram acompanhamento. Além disso as unidades em que havia intervenções por farmacêuticos tiveram reduções nos custos, tanto da unidade como para os próprios pacientes (BOCK et al., 2010).

Cada paciente reage de uma diferente às abordagens, portanto é necessário que o profissional realize o aconselhamento de forma delicada que envolva empatia com perguntas abertas e reflexivas sem que haja um confronto direto ou uma oposição com suas ideias (FRANÇA et al., 2015).

Durante a abordagem, o profissional farmacêutico deve procurar obter o máximo de informações possíveis a respeito da historia de consumo, da motivação, ou de outros assuntos que possam interferir na terapêutica ou na adesão da mesma. O paciente deve ficar ciente sobre 
os riscos de continuar fumando e os benefícios de deixar o vício de lado (CASTELLANO, 2018)

Estando motivado a parar de fumar, deve ser preparado para iniciar o tratamento. O farmacêutico juntamente com o médico deve elaborar um plano terapêutico de acordo com as necessidades do paciente, estabelecendo datas, objetivos, metas terapêuticas, resultados esperados e etc. Deve-se manter o contato com o paciente marcando encontros rotineiros para monitorar a adesão terapêutica para novas orientações ou para alertar o mesmo sobre possíveis efeitos desagradáveis relacionados com a interrupção da prática tabágica ou efeitos adversos importantes (WOOLRYCH; PATERSON; TAN, 2013).

\section{CONCLUSÃO}

Pode se concluir com a realização deste trabalho, que é muito importante o conhecimento sobre os aspectos gerais do tabagismo, sobre os aspectos farmacológicos, sobre as políticas públicas e sobre o tratamento de cessação tabágica. Sendo o tabagismo um problema de saúde pública, necessita-se de pesquisas sobre a temática que possam servir como referência para o embasamento de novos estudos e novas discussões sobre a temática. Portanto este trabalho contribui com o fortalecimento do acervo literário, pois, por se tratar de uma política em construção, ainda são escassas as produções sobre os limites e potencialidades dessa política no Brasil.

\section{REFERÊNCIAS BIBLIOGRÁFICAS}

ADHIKARI, D. et al. A Discussion about Modalities of Smoking Cessation in Perioperative Phase for Addicts: A Review Article. Journal of Addiction Medicine on Therapeutic Science, v. 1, n. 1, p. 102-110, 2014.

AVEYARD, P. et al. Nicotine preloading for smoking cessation: the preloading RCT. Health technology assessment, v. 22, n. 41, p. 1-84, 2018.

BARAONA, L. K. et al. Tobacco harms, nicotine pharmacology, and pharmacologic tobacco cessation interventions for women. Journal of Midwifery \& women's health, v. 62, n. 3, p. 253-269, 2017.

BARBOZA, J. L. et al. An update on the pharmacotherapeutic interventions for smoking cessation. Expert opinion on pharmacotherapy, v. 17, n. 11, p. 1483-1496, 2016. 
BARBOZA, J. L. Pharmaceutical strategies for smoking cessation during pregnancy. 2018. Disponível em: < https://www.tandfonline.com/doi/abs/10.1080/14656566.2018.1538353> Acesso em: 02 dez. 2018.

BENEZECH, B. B.; CHAMPANET, B.; ROUZAUD, P. Smoking cessation at the pharmacy: feasibility and benefits based on a French observational study with six-month follow-up. Substance abuse and rehabilitation, v. 2018, n. 9, p. 31-42, 2018.

BOCK, B. et al. A Tailored intervention to support pharmacy-based counseling for smoking cessation. Nicotine\&tobacco Research, v. 12, n. 3, p. 217-225, 2010.

BRASIL. Ministério da Saúde. Instituto Nacional do Câncer. Comissão Nacional Para implatação da Convenção-Quadro para controle do tabaco (Conicq). Rio de Janeiro. 2015. 62f. Disponível em:

<http://www1.inca.gov.br/inca/Arquivos/comunicacao/convencao_quadro_para_controle_do_ tabaco_texto_oficial.pdf> Acesso em: 25 out. 2018.

BRUNTON, L. L.; CHABNER, B. A.; KNOLLMANN, B. C. As bases farmacológicas da terapêutica de Goodman e Gilman. 12. ed. Porto Alegre: AMGH, 2012.

CAPPELLETTI, K. Redução de tabagismo: projeto de intervençãoem unidade básica de saúde. 2017. 40f. Trabalho de Conclusão de Curso (Pós-graduação em saúde da família) Universidade Federal de Ciências da Saúde de Porto Alegre - UFCSPA, Porto Alegre, 2017. Disponível em: < https://ares.unasus.gov.br/acervo/handle/ARES/9190> Acesso em: 05 out. 2018.

CASTELLANO, M. V. C. O. Abrindo as janelas de oportunidade para tratar o tabagismo. Jornal Brasileiro de Pneumologia, v. 44, n. 3, p. 178-179, 2018.

CORONADO, N. G. et al. Current and emerging pharmacotherapies for cessation of tobacco smoking. The journal of human. Pharmacology and drug therapy, v. 38, n. 2, p. 235-258, 2018.

COSTA, S. C. R. et al. Revisão bibliográfica: políticas públicas do tabagismo no Brasil. Jornal de Ciências da Saúde HU-UFPI, v. 1, n. 2, p. 97-104, 2018.

DANTAS, D. R. G. et al. Tratamento do Tabagismo no Brasil, com bupropiona ou vareniclina: uma revisão sistemática. Revista de saúde e ciências, v. 5, n. 1, p. 61-75, 2016.

ERVILHA, R. R. Avaliação dos fatores facilitadores e dificultadores da cessação do uso do tabaco através de um serviço de intervenção mediado por internet. 2018. 66f. Trabalho de Conclusão de Curso (Curso de Mestrado em saúde coletiva) - Universidade Federal de Juiz de Fora, Juiz de Fora, 2018. Disponível em:

Revinter, v. 12, n. 02, p. 66-83, jun. 2019. 
<http://repositorio.ufjf.br:8080/xmlui/bitstream/handle/ufjf/6856/rafaelarussiervilha.pdf> Acesso em: 27 nov. 2018.

ERWIN, B. L.; SLATON, R. M. Varenicline in the treatment of alcohol use disorders. Annals of pharmacotherapy, v. 48, n. 11, p. 1445-1455, 2014.

FERREIRA, D. A. J. Uso de tabaco e dependência de nicotina em idosos: uma revisão integrada. 2014. 17f. Trabalho de Conclusão de Curso (Especialização em Atenção Básica em saúde da família) - Universidade de Brasília, Brasília, 2014. Disponível em: < http://bdm.unb.br/handle/10483/10779> Acesso em: 05 out. 2018.

FILHO, A. D. de O. et al. The 8-item Morisky Medication Adherence Green Scale: validation of a Brazilian-Portuguese version in hypertensive adults. Elsevier, v. 10, n. 3, p. 554-561, 2014.

FOROUZANFAR, M. H. et al. Global, regional, and national comparative risk assessment of 79 behavioural, environmental and occupational, and metabolic risks or clusters of risks, 1990-2015: a systematic analysis for the Global Burden of Disease Study 2015. The Lancet, v. 388, n.10053, p. 1659-1724, 2016.

FRANÇA, S. A. S. et al. Fatores associados à cessação do tabagismo. Revista de Saúde pública, v. 49, n. 10, p. 2-8, 2015.

GARCIA, T. et al. Avaliação de um tratamento para cessação do tabagismo iniciado durante a hospitalização em pacientes com doença cardíaca ou doença respiratória. Jornal Brasileiro de Pneumologia, v. 44, n. 1, p. 42-48, 2018.

GOLAN, D. E. et al. Princípios de farmacologia: a base fisiopatológica da farmacoterapia. 3. ed. Rio de Janeiro: Guanabara Koogan, 2014.

INSTITUNO NACIONAL DO CÂNCER. Número de fumantes no Brasil cai de $\mathbf{3 0 , 7 \%}$ nos últimos nove anos. INCA. 2015. Disponível em:

$<$ http://www2.inca.gov.br/wps/wcm/connect/agencianoticias/site/home/noticias/2015/numero _fumantes_cai_30_virgula_sete_por_cento_em_nove_anos> Acesso em 02 set. 2018.

INSTITUNO NACIONAL DO CÂNCER. Prevalência de tabagismo. INCA. 2018. Disponível em:

<http://www2.inca.gov.br/wps/wcm/connect/observatorio_controle_tabaco/site/home/dados_ numeros/prevalencia-de-tabagismo> Acesso em 02 set. 2018.

LEMES, E. O. et al. Análise do programa de controle do tabagismo do Ministério da Saúde. Uniciências, v. 21, n. 2, p. 86-92, 2017. 
MAGALHÃES, J. Z.; CAMARGO, E. L. R. A.; SPINOSA, H. S. Vareniclina: uma revisão na perspectiva da promoção da saúde. Caderno de Pós-Graduação em Distúrbios do Desenvolvimento, v. 16, n. 2, p. 55-67, 2016.

MARTINS, E. T. J. Efeitos da cessação do tabagismo na alteração ponderal: estudo coorte prospectiva de vida real. 2016. 59f. Trabalho de Conclusão de Curso (Curso de doutorado em medicina e ciências da saúde) - Universidade Católica do Rio Grande do Sul, Porto Alegre, 2016. Disponível em:

<http://tede2.pucrs.br/tede2/bitstream/tede/7530/2/TES_EDNA_THAIS_JEREMIAS_MART INS_PARCIAL.pdf> Acesso em: 30 nov. 2018.

MARTINS, J. B.; CAPELA, J. P. S.Abordagem farmacológica na cessação tabágica em farmácia comunitária. Revista da Faculdade de ciências da saúde, v. 1, n. 7, p. 246-257, 2010.

MENDES, A. C. R. et al. Custos do programa de tratamento do tabagismo no Brasil. Revista de saúde pública, v. 50, n. 10, 2016. Disponível em:<http://www.scielo.br/scielo.php?pid=S0034-

89102016000100245\&script=sci_arttext\&tlng=pt $>$ Acesso em: 20 out. 2011.

MONTEIRO, C. A. et al. Population-based evidence of a strong decline in the prevalence of smokers in Brazil (1989-2003). Bulletin of the World Health Organization, v. 85, n. 7, p. 527-34, 2007.

MORAES, A. A. A importância dos grupos de apoio para vencer o tabagismo. 2015. 25f. Trabalho de Conclusão de Curso (Curso de Especialização em Atenção básica e saúde da família) - Universidade Federal do Mato Grosso do Sul, Campo Grande, 2015. Disponível em: < https://ares.unasus.gov.br/acervo/handle/ARES/10307> Acesso em: 25 out. 2018.

MOURA, C. F. Uma abordagem multiprofissional em unidade básica de saúde contra o tabagismo. 2014. 18f. Trabalho de Conclusão de Curso (Curso de Especialização em Atenção Básica e Saúde da Família) - Universidade Federal de Mato Grosso do Sul, Goianira, 2014. Disponível em: <https://ares.unasus.gov.br/acervo/handle/ARES/3558> Acesso em: 27 nov. 2018.

NUNES, B. M. R. et al. Atenção farmacêutica no contexto do tratamento de tabagistas: estudo de caso. Biofarma, v. 13, n. 2, p. 21-28. 2017.

OBERG, M. et al. Worldwide burden of disease from exposure to second-hand smoke: a retrospective analysis of data from 192 countries. The Lancet, v. 377, n. 9760, p. 139-146, 2011.

PEREIRA, A. A. C. et al. Adesão ao grupo de cessação entre tabagistas de unidade básica de saúde. Cogitare Enfermagem, v. 23, n. 3, 2018. Disponível em: <

http://www.saude.ufpr.br/portal/revistacogitare/wp-content/uploads/sites/28/2018/08/55096239422-1-PB.pdf> Acesso em 15 out. 2018.

Revinter, v. 12, n. 02, p. 66-83, jun. 2019. 


\section{Revinter}

PINTO, M. et al. Carga de doença atribuível ao uso do tabaco no Brasil e potencial impacto do aumento de preços por meio de impostos. Documento técnico. 2017. Disponível em:

<http://actbr.org.br/uploads/arquivo/1173_Doc_Tec_Brasil_fi_al_plain_portugues_24-517.pdf> Acesso em: 26 out. 2018.

PINTO, M. T.; RIVIERE, A. P.; BARDACH, A. Estimativa da carga do tabagismo no Brasil: mortalidade, morbidade e custos. Cad. Saúde Pública. v.31, n.6, p. 1283-1297, 2015.

Disponível em: <http://www.scielo.br/pdf/csp/v31n6/0102-311X-csp-31-6-1283.pdf> Acesso em 20 out. 2018.

PORTES, L. H. et al. A política de controle do tabaco no Brasil: Um balanço de 30 anos. Ciências \& saúde coletiva, v. 23, n. 6, p. 1837-1848, 2018.

PURPULIM, A. F. et al. Mecanismos de depêndencia química no tabagismo: revisão da literatura. Revista médica da Universidade Federal do Paraná, v. 2, n. 2, p. 74-78, 2015.

RABAHI, M. F.; ALCÂNTARA, E. C. Tendência temporal da endemia do tabagismo no Brasil. Revista médica de Minas Gerais, v. 25, n. 1, p. 140-142, 2015.

ROLLEMBERG, E. V. et al. Abordagem terapêutica para cessação do tabagismo em esquizofrênicos: uma revisão de literatura. Revista Medicina e Saúde de Brasília, v. 6, n. 3, p. 359-371, 2017.

SANTANA, K. dos S. etal. O papel do profissional farmacêutico na promoção da saúde e do uso racional de medicamentos. Revista FAEMA, v. 9, n. 1, p. 399-412, 2018.

SILVA, G. P. Tabagismo na UBS Antônio Afonso Magalhães em Sarzedo - uma mudança de perspectiva. 2015. 33f. Trabalho de Conclusão de Curso (Graduação em Enfermagem) - Universidade Federal de Minas Gerais, Minas Gerais, 2015. Disponível em: < https://ares.unasus.gov.br/acervo/handle/ARES/8920> Acesso em: 27 nov. 2018.

SILVA, L. C. C. et al. Smoking control: challengs and achievements. Jornal brasileiro de pneumologia, v. 42, n. 4, p. 290-298, 2016.

SILVA, S. T. et al. Combate ao tabagismo no Brasil: a importância estratégica das ações governamentais. Ciências \& saúde coletiva, v. 19, n. 2, p. 539-552, 2014.

SOARES, F. dos S. et al. Prevalência de tabagismo e doença pulmonar obstrutiva crônica em doentes arteriais periféricos: resultados preliminares. Federation International of Physical Education Bulletin, v. 84, n. 2, 2014. Disponível em: < http://www.fiepbulletin.net/index.php/fiepbulletin/article/viewFile/4626/9050> Acesso em 02 nov. 2018. 
WOLD HEALTH ORGANIZATION. Tobacco. Disponível em: <http://www.who.int/newsroom/fact-sheets/detail/tobacco> Acesso em 29 ago. 2018.

WOOLRYCH M. H.; PATERSON, H.; TAN, M. Exposure to the smoking cessation medicine varenicline during pregnancy: a prospective nationwide cohort study.

Pharmacoepidemiology Drug Safety, v. 22, n. 10, p. 1086-1092, 2013. 